\title{
Stochastic Resonance within the Somatosensory System: Effects of Noise on Evoked Field Potentials Elicited by Tactile Stimuli
}

\author{
Elías Manjarrez, Gerardo Rojas-Piloni, Ignacio Méndez, and Amira Flores \\ Instituto de Fisiología, Benemérita Universidad Autónoma de Puebla, Puebla, Pue CP 72570, México
}

\begin{abstract}
Stochastic resonance (SR) is commonly understood to be the enhancement, by noise, of the response of a system to a weak input signal. The aim of this study was to demonstrate the occurrence of SR in spinal and cortical evoked field potentials (EFPs) elicited by periodic tactile stimuli in the anesthetized cat. The electrodes were positioned in spinal and cortical somatosensory regions in which the largest negative EFPs were detected. The periodic tactile stimuli consisted of local skin displacements on the central pad of the hindpaw. Two series of experiments were performed. First, periodic tactile stimuli and the noisy tactile stimuli were applied with the same indenter. Second, noisy tactile stimuli were applied with an additional indenter placed on the glabrous skin of the third hindpaw digit. This last protocol ensured that the signal and noise were mixed not in the skin but in the somatosensory regions of the CNS. All cats showed distinct SR behavior at the spinal and cortical stages of the sensory encoding. Such SR was abolished in the cortical but not in the spinal recording after sectioning of the dorsal columns and the ipsilateral dorsolateral funiculus. This suggests that the spinal neurons may also contribute to the SR observed at the cortical level. To the best of our knowledge, this is the first documented evidence that such a remarkable phenomenon embodies electrical processes of the spinocortical somatosensory system itself.
\end{abstract}

Key words: noise; tactile; information capacity; stochastic resonance; somatosensory; evoked potentials

\section{Introduction}

Stochastic resonance (SR) is a counterintuitive phenomenon of nonlinear systems that refers to the increase of the signal-to-noise ratio (SNR) on the output obtained through an increase of the noise level on the input (Wiesenfeld and Moss, 1995; Gammaitoni et al., 1998; Anishchenko et al., 1999). Typically, the plot of SNR versus input noise is an inverted U-like function characterized by maximal enhancement of SNR at a specific noise amplitude value. SR type effects have been demonstrated in physical and biological systems (Segundo et al., 1994; Gammaitoni et al., 1998; Anishchenko et al., 1999; Russell et al., 1999; Winterer et al., 1999; Hidaka et al., 2000, 2001; Stacey and Durand, 2000; Hanggi, 2002; Manjarrez et al., 2002c,d; Mori and Kai, 2002; Ward et al., 2002; Yamamoto et al., 2002).

The SR has been shown in psychophysical experiments of cutaneous tactile sensation in humans. These studies have shown that the presence of a particular nonzero level of noise may significantly enhance the ability of an individual to detect subthreshold tactile stimuli (Collins et al., 1996b, 1997; Richardson et al., 1998; Dhruv et al., 2002; Liu et al., 2002). Furthermore, recent electrophysiological evidence in humans suggests that the cortical neurons that participate in the process also exhibit SR

Received 0ct. 17, 2002; revised Dec. 23, 2002; accepted Dec. 27, 2002.

This work was supported in part by Consejo Nacional de Ciencia y Tecnología Grant J36062-N (E.M.) and by grants from Fondo-Ricardo J. Zevada (A.F.) and Fondo para Modernizar la Educación Superior-Benemérita Universidad Autónoma de Puebla, México.

Correspondence should be addressed to Dr. Elías Manjarrez at the above address. E-mail: emanjar@siu.buap.mx. Copyright $\odot 2003$ Society for Neuroscience $\quad 0270-6474 / 03 / 231997-05 \$ 15.00 / 0$
(Manjarrez et al., 2002c). However, it is not clear from these experiments whether the electrical activity of the spinal neurons also exhibits SR behavior or whether the SR is produced only in the peripheral sensory receptors. In this context, the purpose of the present study was to substantiate whether SR occurs in the spinal and cortical somatosensory system itself in a preparation of the anesthetized cat. Disclosure of this phenomenon in the spinal and cortical stages of the sensory encoding could be important, because a major goal of contemporary studies of sensory processing is to understand the transformations of signals at each level within the CNS.

\section{Materials and Methods}

Preparation. Experiments were performed in 10 adult cats (weight range, $2.0-3.5 \mathrm{~kg})$ initially anesthetized with pentobarbitone $(35 \mathrm{mg} / \mathrm{kg}$, i.p.). Most procedures have been described previously (Manjarrez et al., 2002a,b,d) and are briefly described here. Guidelines contained in the National Institutes of Health Guide for the Care and Use of Laboratory Animals (85-23, revised in 1985) were followed throughout.

First protocol of stimulation. Periodic tactile stimuli and noisy tactile stimuli were applied with the same indenter placed on the glabrous skin of the central pad of the hindpaw (Fig. 1A). The indenter consisted of a closed-loop mechanical stimulator-transducer that allowed measures of the force and displacement of applied stimuli. The output of two independent function generators provided input to the stimulator-transducer. One of these [Tektronix (Wilsonville, OR) CFG253 together with AMPI (Jerusalem, Israel) Master-8] generated the test stimulus signal, whereas the other [Wavetek (San Diego, CA) 132] supplied the superimposed noise. 
A
INPUT

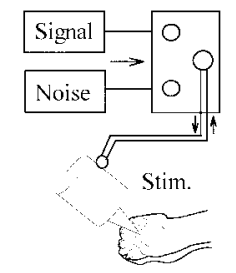

B

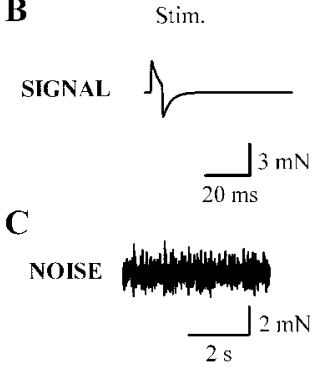

D

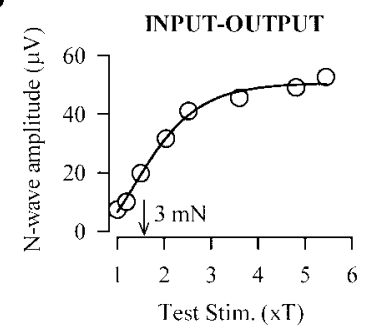

OUTPUT
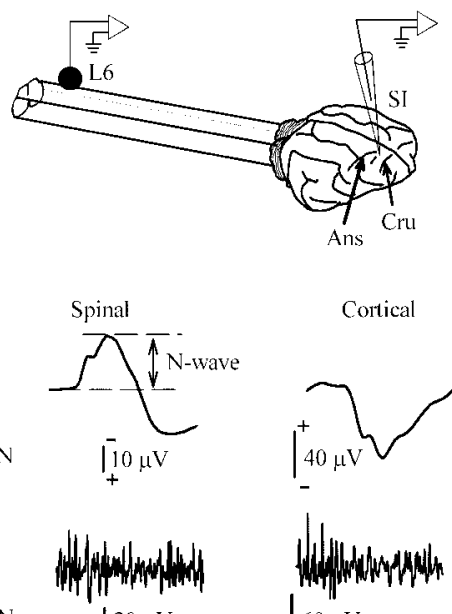

$120 \mu \mathrm{V}$

$\mathbf{E}$

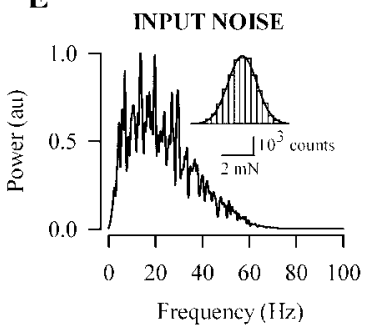

Figure 1. $A$, Scheme of the experimental arrangement. For the first protocol, the periodic and noisy tactile stimuli were applied with the same indenter. $B$, Records of the input signal and the spinal and cortical evoked potentials. $C$, Records with the same format as that in $B$ : input noise alone and the simultaneously recorded spinal and cortical activity (output) for one level of noise, $\sigma_{n}=1.2 \mathrm{mN}$. D, Mean N-wave amplitude of the spinal cord dorsum potential versus test stimulus strength. E, Power spectrum of the input noise illustrated in $C$. The inset in $E$ shows the amplitude distribution of the input noise. Ans, Ansate sulcus; au, arbitrary units; $C r u$, cruciate sulcus; Stim., stimulus; $X T$, times threshold.

Second protocol of stimulation. Noisy tactile stimuli were applied with an additional indenter placed on the glabrous skin of the third hindpaw digit, whereas the periodic tactile stimuli were applied on the glabrous skin of the central pad of the hindpaw (see Fig. $3 A$ ). This protocol ensured that the signal and noise were mixed not in the skin but in the somatosensory regions of the CNS (see also Mori and Kai, 2002).

To avoid possible peripheral mixing of the noisy stimuli and the periodic stimuli caused by the elasticity of the adjoining skin, the hindlimb and the third hindpaw digit were held in a fixed position. With this procedure, no evidence of mixing was detected with the mechanical transducers of both stimulators.

Test stimuli (input signal). Mechanical test stimuli (local skin displacements) were applied on the central pad of the hindpaw. Such stimuli consisted of single pulses with a total duration of $10 \mathrm{msec}$ delivered at a constant frequency of $2.5 \mathrm{~Hz}$ (Fig. $1 \mathrm{~B}$ ). We assumed that the SR might be present at this frequency because at this same frequency of tactile stimulation, we observed the SR in human brain waves (Manjarrez et al., 2002c). Figure $1 B$ illustrates typical spinal and cortical evoked field potentials (EFPs) elicited by the test stimulus. In the present experiments, the amplitude of the test stimulus was adjusted to $3 \mathrm{mN}$, between 1.2 and 1.5 times the threshold level of the afferent volley recorded on the surface of the spinal cord. We assumed that the force of $3 \mathrm{mN}$ would be sufficient to activate slowly adapting and fast-adapting mechanoreceptive afferents innervating the skin (Trulsson, 2001). Figure $1 D$ shows the input-output curve for the spinal $\mathrm{N}$-wave amplitude at L6 versus the test stimulus strength.

Input noise. Noise with a power spectrum ranging from 0.1 to $60 \mathrm{~Hz}$ (Fig. $1 E$ ) was applied on the central pad of the hindpaw (in the first protocol) and on the third hindpaw digit (in the second protocol). The range of the $\mathrm{SD}$ of the noisy stimuli $\left(\sigma_{n}\right)$ was from 0.1 to $5 \mathrm{mN}$. Figure $1 E$ shows the power spectrum of a typical noisy stimulus of $\sigma_{n}=1.2 \mathrm{mN}$. The inset in Figure $1 E$ shows the amplitude distribution (Gaussian) of the noisy stimulus.

Stimulation scheme. The stimulation scheme consisted of sequences that lasted $20 \mathrm{sec}$, during which we applied either a periodic stimulus (signal) with noise superimposed (Fig. $2 A$, insets, red traces) or noise alone (Fig. $2 A$, insets, blue traces). We applied 10 sequences, each with a different noise intensity level. For example, insets in Figure $2 \mathrm{~A}$ illustrate some sequences from the first protocol. The presentation order of the different noise levels was varied randomly to remove possible serial effects.

Electrophysiological recordings. In the experiments with the first protocol, spinal potentials were recorded from the surface of the L6 dorsal horn with a silver ball electrode against an indifferent electrode placed on the near paravertebral muscles (Fig. $1 \mathrm{~A}$ ). In addition, one glass micropipette filled with $1.2 \mathrm{M} \mathrm{NaCl}$ (tip diameter, $1.0-2.5 \mu \mathrm{m} ; 1.2-1.7 \mathrm{M} \Omega$ ) was used to record cortical EFPs in the right posterior sigmoid gyrus (layers III-V). The micropipette entered the hindlimb representation of the primary somatosensory cortex (S1) (Figs. $1 A, 3 A, C$ ) located in the posterior sigmoid gyrus (Felleman et al., 1983). In the experiments with the second protocol, we recorded the spinal EFPs with an additional micropipette inserted in the dorsal horn (Fig. 3A,B). The micropipette was positioned in the intraspinal depth, where the negative EFPs acquired their maximal amplitude (laminas III-VI) (Fig. 3B).

Data analysis. Data acquisition of the input noise and of the spinal and cortical potentials was performed with a sampling rate of $500 \mathrm{~Hz}$. Spectral analysis of spinal and cortical activity recorded during each of the 20 sec stimulation epochs was performed. The magnitude of the input noise was quantified by means of the SD of the input force ( $\sigma_{n}$ of input noise). We estimated the effect of noise on spinal and cortical EFPs from the output power spectra (Fig. $2 B, C$ ). SNR (Fig. $2 D$ ) was defined as the ratio, at the input signal frequency $(2.5 \mathrm{~Hz})$, of the strength of the output power spectra peak (its area) during pulse stimulation to the mean amplitude of the output power spectra that occurred during input noise alone. The method to calculate SNR has been described in detail previously (Manjarrez et al., 2002c). In addition, information capacity (Shannon, 1949) at the spinal and cortical stages of the sensory encoding was calculated. Information capacity $\left(R_{\text {info }}\right)$ is the maximum achievable rate of information transmission through a communication system in bits per second (Fig. 2D). For comparison, we performed an identical analysis of the signals before and after section of the dorsal columns (DC) and ipsilateral dorsolateral funiculus (IDLF).

Histology and verification of electrode placements. At the end of the experiment, each animal was killed with a pentobarbitone overdose and perfused with $10 \%$ formalin. The spinal cord and the brain were removed, and the recording micropipettes were left in place. After complete fixation and dehydration, both the spinal cord and the brain were placed in a solution of methyl salicylate for clearing and subsequently cut so that both sections contained the electrodes (Fig. 3B,C) and lesions in the T12 segment (Fig. $4 F$ ) of the spinal cord.

\section{Results}

First protocol: periodic tactile stimuli and noisy tactile stimuli applied with the same indenter

All animals we examined exhibited clear-cut SR-type behavior elicited by tactile stimulation on spinal and cortical activity. Figure $2 E, F$ (circles) illustrates the output SNR at the two stages of sensory encoding, spinal (Fig. 2 E, open circles) and cortical (Fig. $2 F$, filled circles), versus $\sigma_{n}$ for one cat. In all cases, the test stimulus signal was adjusted to $3 \mathrm{mN}$, and the range of the noisy tactile stimuli was from $\sigma_{n}=0.1-5 \mathrm{mN}$. An average of the spinal and cortical SNR peaks was calculated for all animals (8.7 \pm 4.2 and $5.5 \pm 3.8$, respectively; mean $\pm \mathrm{SD} ; n=6$ cats). Similarity in the inverted $\mathrm{U}$-like feature of the graphs calculated from each of the cats was such that a qualitative general description of these curves may apply to all. As the noise amplitude increased, SNR values 


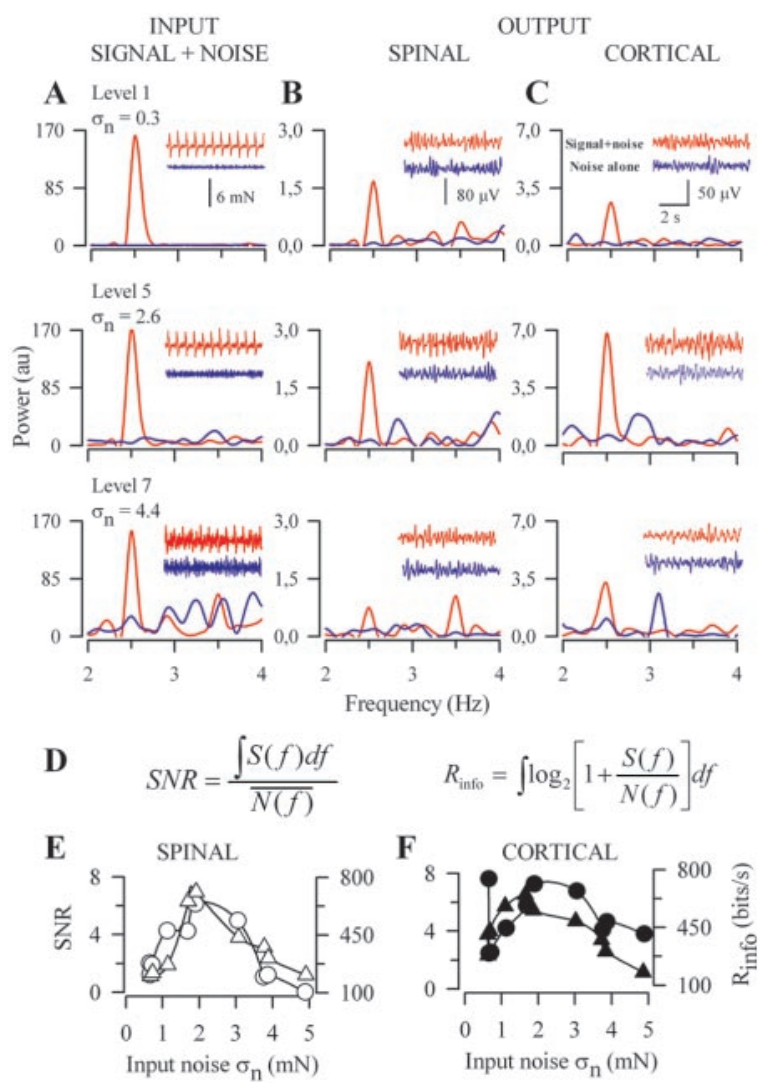

Figure 2. A, Representative power spectra of the input periodic signal plus noise (traces in red) and noise alone (traces in blue) for three different levels of noise. In each case, $\sigma_{n}$ indicates the SD of the input noise. $B, C$, Plots with the same format as that in $A$ but for the simultaneously recorded spinal and cortical activity elicited by the stimuli illustrated in the insets of $2 A$. The insets in $A-C$ show typical recordings from which the power spectra were calculated. D, Formulas of SNR and $R_{\text {info }} . S(f)$ corresponds to the power spectrum of the output neuronal activity (spinal or cortical) elicited by the periodic stimuli $(2.5 \mathrm{~Hz})$ plus noise. $N(f)$ is the power spectrum of the output neuronal activity (spinal or cortical) elicited by noise alone. E, F, Output SNR (circles) and $R_{\text {info }}$ (triangles) versus $\sigma_{n}$ for one cat. E, Spinal (open symbols) output SNR and $R_{\text {info }}$ versus $\sigma_{n}$. F, Cortical ( filled symbols) output SNR and $R_{\text {info }}$ versus $\sigma_{n}$. These results were obtained from experiments with the first protocol. au, Arbitrary units.

became larger. Hence, a positive slope and an upsurge of the function could be observed as the curve rose steeply and became convex. A maximum value of SNR was reached, and the slope became zero within a particular interval of noise amplitudes. Beyond such peak, with higher noise amplitudes, the slope became negative as the curve subsided gradually. This modulation of the SNR suggests quite strongly that the information transmitted through the somatosensory system (i.e., $R_{\text {info }}$ ) was also modulated by the input noise. Figure $2 E, F$ (triangles) illustrates the $R_{\text {info }}$ at the two stages of sensory encoding, spinal (Fig. $2 E$, open triangles) and cortical (Fig. $2 F$, filled triangles), versus $\sigma_{n}$ for one cat. These plots also were typical of SR-like phenomena. An average of the spinal and cortical $R_{\text {info }}$ peaks was calculated for all animals (593.6 \pm 128.8 and $573.2 \pm 185.3 \mathrm{bits} / \mathrm{sec}$, respectively; mean $\pm \mathrm{SD} ; n=6$ cats $)$.

\section{Second protocol: noisy tactile stimuli applied with an additional indenter placed on the glabrous skin of the third hindpaw digit}

In three other cats, the noisy tactile stimulus was applied with another indenter placed on the glabrous skin of the third hindpaw digit (Fig. 3A). This experimental arrangement ensured that
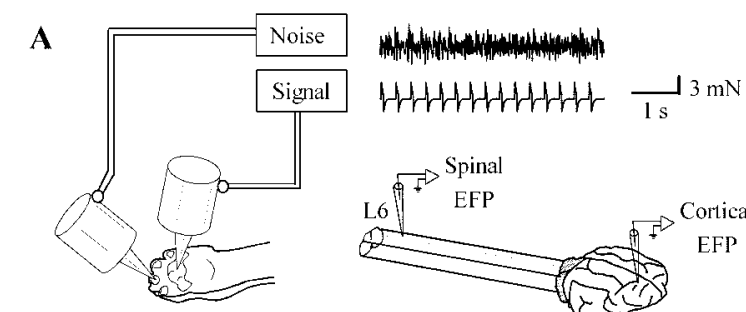

B

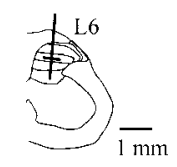

C
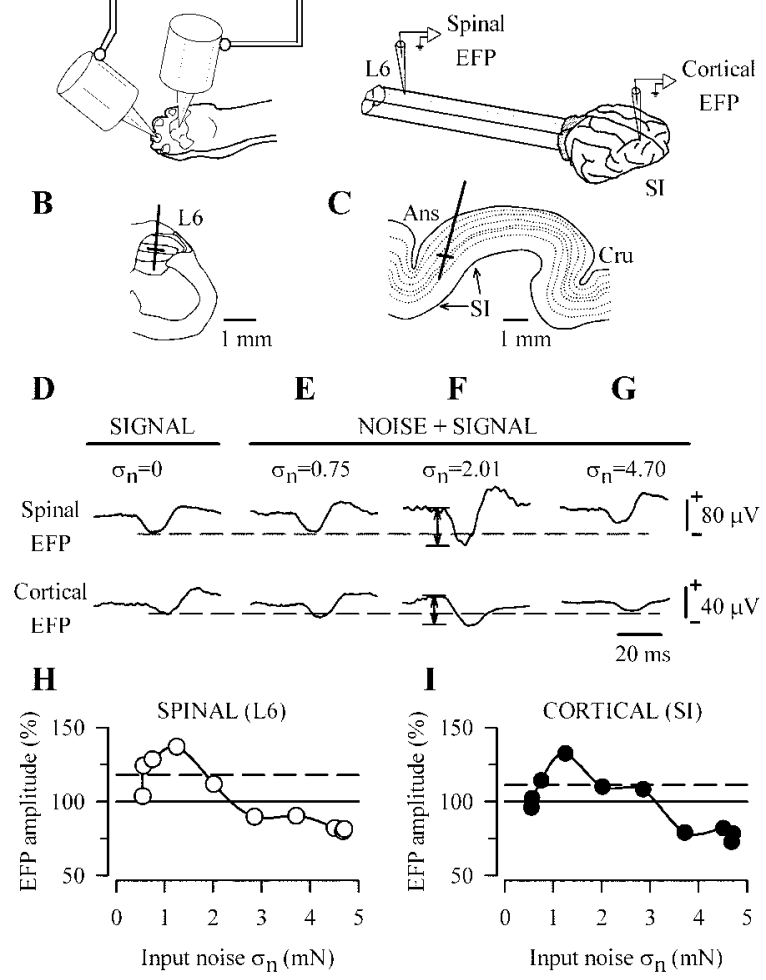

F $\quad$ G

NOISE + SIGNAL
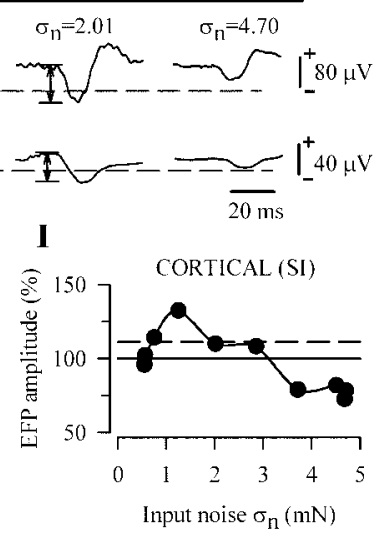

Figure 3. A, Scheme of the experimental arrangement. For the second protocol, the noise and signal stimuli arrive at the dorsal horn via separate pathways. This protocol ensured that the signal and noise were mixed not in the skin but in the somatosensory regions of the CNS. The electrodes were positioned in spinal and cortical somatosensory regions in which the largest EFPs were detected. Drawings in $B$ and $C$ are histological reconstructions of the electrode tracks within the dorsal horn at $L 6$ and within the $S 1$ somatosensory cortex. $D-G$, Averages $(n=32)$ of spinal and cortical EFPs recorded at intraspinal and intracortical depths, as indicated in $B$ and C.D, Averages of spinal and cortical EFPs in control conditions $\left(\sigma_{n}=0\right)$. $E-G$, Averages of spinal and cortical EFPs for three different levels of noise (i.e., signal plus noise, $\sigma_{n}=0.75, \sigma_{n}=2.01$, $\left.\sigma_{n}=4.7\right)$. H, Facilitation of spinal EFPs (amplitude) versus $\sigma_{n}$ for one experiment. The dashed line represents the magnitude of a $95 \%$ confidence interval. Amplitude of EFPs was measured as indicated by the arrows in F. I, The same as $H$ but for cortical EFPs. Ans, Ansate sulcus; $\mathrm{Cru}$, cruciate sulcus.

the signal and noise were mixed not in the skin but in the somatosensory regions of the CNS. The traces in Figure 3D-G show averages of the spinal and cortical EFPs produced by periodic tactile stimuli on the glabrous skin of the central pad of the hindpaw. The spinal EFPs were recorded at a depth of $1250 \mu \mathrm{m}$ within the dorsal horn, and the cortical EFPs were recorded at a depth of $1400 \mu \mathrm{m}$ within the S1 somatosensory cortex. In these experiments, we examined the effects of noise (applied on the glabrous skin of the third hindpaw digit) on the amplitude of the spinal and cortical EFPs produced by periodic tactile stimuli (applied on the glabrous skin of the central pad of the hindpaw). Figure $3 H, I$ illustrates plots of percentage changes of EFP amplitude relative to control (taken as $100 \%$ ) versus $\sigma_{n}$ for one experiment. In these plots, the control was considered as the response to the test stimulus alone (Fig. 3D). Note that each plot (Fig. 3H,I) exhibited a maximal enhancement of EFP amplitude at a specific noise amplitude value (i.e., the spinal and cortical EFPs were facilitated within a particular interval of noise amplitudes). An average of the spinal and cortical facilitation peaks was calculated for all 


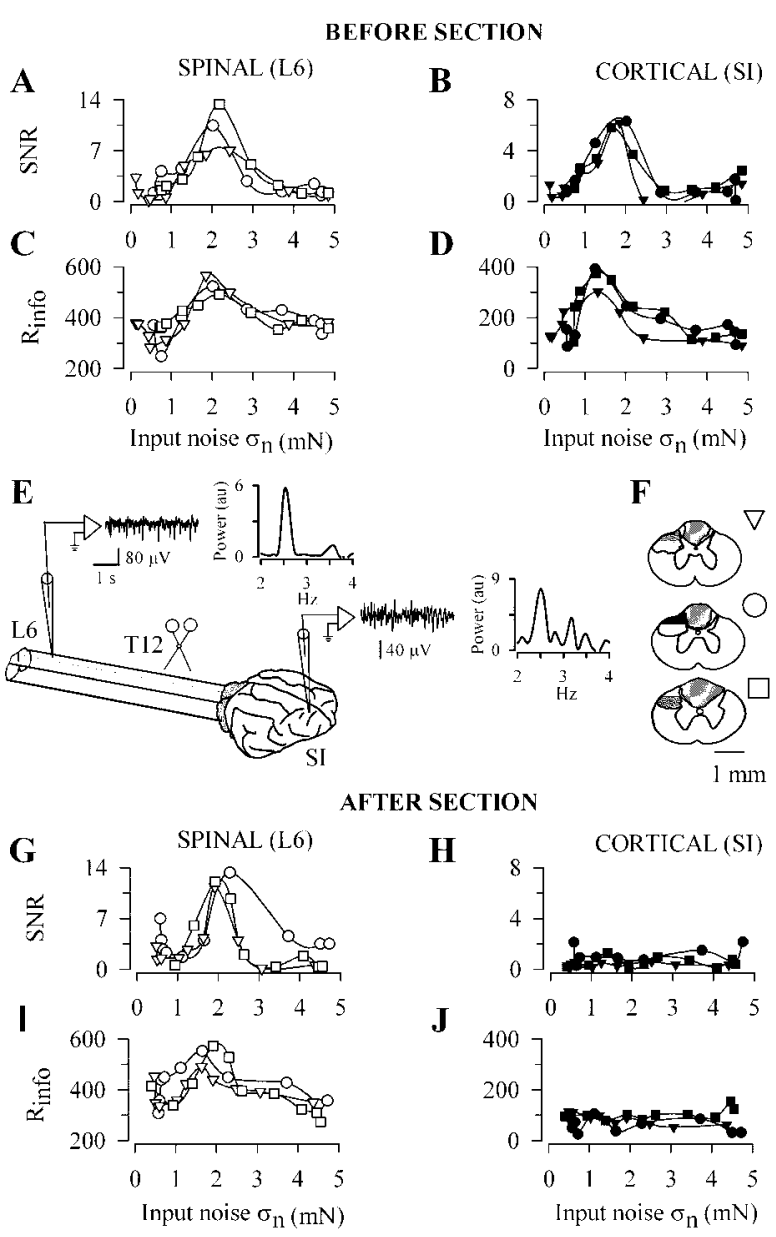

Figure 4. Spinal $(A)$ and cortical ( $B)$ output SNR versus $\sigma_{n}$ for three different cats (indicated by different symbols) in control conditions (before section). $G, H$, The same as $A$ and $B$, but after section (at T12) of the DC and the IDLF illustrated in $E$ and $F$. F, Drawings from the histological sections of the DC and IDLF at T12 obtained from three cats (indicated by different symbols). $C$ and $D$ and $/$ and $J$ are the same as $A$ and $B$ and $G$ and $H$ but for $R_{\text {info }}$. Note that $S R$ disappears in the cortical (filled symbols) but not in the spinal (open symbols) stages after section. These results were obtained from experiments with the second protocol.

animals $[120.3 \pm 10.3 \%(n=4)$ and $124.1 \pm 15.1 \%(n=3)$, respectively; mean $\pm \mathrm{SD}$ ].

Furthermore, all of the animals we examined with the second protocol exhibited SR-type behavior elicited by tactile stimulation on spinal and cortical activity. Figure 4 was obtained from three cats (indicated by different symbols). Figure $4 A-D$ illustrates the output SNR (and $R_{\text {info }}$ ) at the two stages of sensory encoding, spinal (Fig. 4A,C, open symbols) and cortical (Fig. $4 B, D$, filled symbols), versus $\sigma_{n}$ in control conditions. Figure $4 E$ illustrates typical recordings and power spectra obtained from the spinal and cortical evoked potentials.

\section{SR after sectioning of DC and IDLF}

The SR behavior was abolished in the cortical (Fig. 4H,J) but not in the spinal (Fig. 4G,I) recordings after sectioning (Fig. 4E,F) of DC and IDLF. Averages of the cortical SNR and $R_{\text {info }}$ peak values (in the band from 1 to $2 \mathrm{mN}$ ) (Fig. $4 B, D$ ) were calculated for three animals ( $4.8 \pm 1.4$ and $303.5 \pm 68.7 \mathrm{bits} / \mathrm{sec}$, respectively). These averages were different $(p<0.001$; Student's $t$ test) from the averages of the cortical SNR and $R_{\text {info }}$ values $(0.5 \pm 0.5$ and $85.4 \pm 21.3 \mathrm{bits} / \mathrm{sec}$, respectively, in the band from 1 to $2 \mathrm{mN}$ ) (Fig. $4 \mathrm{H}, \mathrm{J}$ ) obtained after the sections illustrated in Figure $4 \mathrm{~F}$.

\section{Discussion}

SR occurs in the spinocortical somatosensory system itself and not only in the peripheral sensory receptors

The SR has been well described in the peripheral sensory system (Douglass et al., 1993; Collins et al., 1996a; Cordo et al., 1996; Levin and Miller, 1996; Juusola and French, 1997; Ivey et al., 1998; Jaramillo and Wiesenfeld, 1998; Bahar et al., 2002). However, it is not clear from these experiments whether the electrical activity of somatosensory neurons in the CNS also exhibits SR behavior or whether SR is produced exclusively in the peripheral sensory receptors.

Our results show that a certain range of noise can increase spinal and cortical EFPs (Fig. 3H,I). This result provides a possible explanation of the SR observed in the SNR and $R_{\text {info }}$ of the spinal and cortical activity evoked by mechanical tactile stimuli (Fig. 4A-D). In particular, we demonstrated that the SR embodies electrical processes of the spinocortical somatosensory system itself (see also Manjarrez et al., 2002d). We used a protocol, as shown in Figure $3 A$, that ensured that the signal and noise were mixed not in the skin but in the somatosensory regions of the CNS. In this context, our results show that the SR occurs in the spinal and cortical somatosensory system itself and not only in the peripheral sensory receptors.

To the best of our knowledge, the present investigation documents the first explicit explanation of the occurrence of SR phenomena concerning the electrical activity of the spinal and cortical stages of sensory encoding in an in vivo preparation. Our results agree well with the psychophysical findings of Collins et al. (1996b, 1997), who described that the ability of an individual to detect a subthreshold tactile stimulus can be enhanced by introducing a particular level of noise. Furthermore, our results are consistent with recent evidence that the SNR of cortical activity elicited by mechanical tactile stimuli in humans is optimized by the presence of noise (Manjarrez et al., 2002c).

Our experiments show that the range of noise intensities necessary for the enhancement of SNR and $R_{\text {info }}$ was within physiological limits $(1-4 \mathrm{mN})$, in the same range in which noise can improve tactile sensation in humans (Collins et al., 1996b, 1997; Manjarrez at al., 2002c). Furthermore, our experiments show that the SR was evident at $2.5 \mathrm{~Hz}$, the same frequency of tactile stimulation at which the SR was observed in the human brain (Manjarrez et al., 2002c). These evidences suggest that noise could play a major physiological role in tactile sensation by somatosensory neurons, both in cats and in humans.

Several causes may explain the different profiles observed in the SNR and $R_{\text {info }}$ graphs obtained from different experiments (Figs. 2E, F , 4A-D). The diversity of these profiles between animals may be attributed to their different sensitivity to stimuli, dissimilarities in skin elasticity, receptor density, and irregularity of the background activity at the spinal, brainstem, thalamic, and cortical levels.

Participation of dorsal horn spinal neurons in the mechanism of generation of SR at the cortical level

Previous work from our laboratory (Manjarrez et al., 2002a,b) has shown that somatosensory cortical neurons can be driven by dorsal horn spinal neurons with spontaneous activity through the DC and the spinocervical tract. The cell bodies of origin of the spinocervical tract are located in the lumbosacral dorsal horn (laminas IV-VI) (Bryan et al., 1973), the same region in which the neurons that produce spontaneous negative cord dorsum potentials are located. These observations show that the first synapse for most tactile afferents is also located within the dorsal horn 
(Fig. 3B). In this context, the present study suggests that such dorsal horn spinal neurons of the spinocervical tract may also contribute to the mechanism of generation of SR at the cortical level. The present results (Fig. 4) are consistent with this possibility, because the SR disappears in the cortical but not in the spinal recording after sectioning of the DC and the IDLF (see also Manjarrez et al., 2002a). We conclude that the SR may occur in the spinocortical somatosensory system itself in a preparation of the anesthetized cat.

\section{References}

Anishchenko VS, Neiman AB, Moss F, Schimansky-Geier L (1999) Stochastic resonance: noise-enhanced order. Physics-Uspekhi 42:7-36.

Bahar S, Neiman A, Wilkens LA, Moss F (2002) Phase synchronization and stochastic resonance effects in the crayfish caudal photoreceptor. Phys Rev E Stat Nonlin Soft Matter Phys 65:050901.

Bryan RN, Trevino DL, Coulter JD, Willis WD (1973) Location and somatotopic organization of the cells of origin of the spino-cervical tract. Exp Brain Res 17:177-189.

Collins JJ, Imhoff TT, Grigg P (1996a) Noise-enhanced information transmission in rat SA1 cutaneous mechanoreceptors via aperiodic stochastic resonance. J Neurophysiol 76:642-645.

Collins JJ, Imhoff TT, Grigg P (1996b) Noise-enhanced tactile sensation. Nature 383:770.

Collins JJ, Imhoff TT, Grigg P (1997) Noise-mediated enhancements and decrements in human tactile sensation. Phys Rev E Stat Nonlin Soft Matter Phys 56:923-926.

Cordo P, Inglis JT, Verschueren S, Collins JJ, Merfeld DM, Rosenblum S, Buckley S, Moss F (1996) Noise in human muscle spindles. Nature 383:769-770.

Dhruv NT, Niemi JB, Harry JD, Lipsitz LA, Collins JJ (2002) Enhancing tactile sensation in older adults with electrical noise stimulation. NeuroReport 13:597-600.

Douglass JK, Wilkens L, Pantazelou E, Moss F (1993) Noise enhancement of information transfer in crayfish mechanoreceptors by stochastic resonance. Nature 365:337-340.

Felleman DJ, Wall JT, Cusick CG, Kaas JH (1983) The representation of the body surface in S-I of cats. J Neurosci 3:1648-1669.

Gammaitoni L, Hanggi P, Jung P, Marchesoni F (1998) Stochastic resonance. Rev Mod Phys 70:223-287.

Hanggi P (2002) Stochastic resonance in biology. How noise can enhance detection of weak signals and help improve biological information processing. Chemphyschem 3:285-290.

Hidaka I, Nozaki D, Yamamoto Y (2000) Functional stochastic resonance in the human brain: noise induced sensitization of baroreflex system. Phys Rev Lett 85:3740-3743.

Hidaka I, Ando S, Shigematsu H, Sakai K, Setoguchi S, Seto T, Hirooka Y, Takeshita A, Yamamoto Y (2001) Noise-enhanced heart rate and sympathetic nerve responses to oscillatory lower body negative pressure in humans. J Neurophysiol 86:559-564.

Ivey C, Apkarian AV, Chialvo DR (1998) Noise-induced tuning curve changes in mechanoreceptors. J Neurophysiol 79:1879-1890.
Jaramillo F, Wiesenfeld K (1998) Mechanoelectrical transduction assisted by Brownian motion: a role for noise in the auditory system. Nat Neurosci 1:384-388.

Juusola M, French AS (1997) The efficiency of sensory information coding by mechanoreceptor neurons. Neuron 18:959-968.

Levin JE, Miller JP (1996) Broadband neural encoding in the cricket cercal sensory system enhanced by stochastic resonance. Nature 380:165-168.

Liu W, Lipsitz LA, Montero-Odasso M, Bean J, Kerrigan DC, Collins JJ (2002) Noise-enhanced vibrotactile sensitivity in older adults, patients with stroke, and patients with diabetic neuropathy. Arch Phys Med Rehabil 83:171-176.

Manjarrez E, Rojas-Piloni G, Vázquez D, Flores A (2002a) Cortical neuronal ensembles driven by dorsal horn spinal neurones with spontaneous activity in the cat. Neurosci Lett 318:145-148.

Manjarrez E, Rojas-Piloni G, Martínez L, Vázquez D, Vélez D, Méndez I, Flores A (2002b) Amplitude of somatosensory cortical evoked potentials is correlated with spontaneous activity of spinal neurones in the cat. Neurosci Lett 323:187-190.

Manjarrez E, Diez-Martínez O, Méndez I, Flores A (2002c) Stochastic resonance in human electroencephalographic activity elicited by mechanical tactile stimuli. Neurosci Lett 324:213-216.

Manjarrez E, Rojas-Piloni G, Méndez I, Martínez L, Vélez D, Vázquez D, Flores A (2002d) Internal stochastic resonance in the coherence between spinal and cortical neuronal ensembles in the cat. Neurosci Lett 326:93-96.

Mori T, Kai S (2002) Noise-induced entrainment and stochastic resonance in human brain waves. Phys Rev Lett 88:1-4.

Richardson KA, Imhoff TT, Grigg P, Collins JJ (1998) Using electrical noise to enhance the ability of humans to detect subthreshold mechanical cutaneous stimuli. Chaos 8:599-603.

Russell DF, Wilkens LA, Moss F (1999) Use of behavioural stochastic resonance by paddle fish for feeding. Nature 402:291-294.

Segundo JP, Vibert JF, Pakdaman K, Stiber M, Diez-Martínez O (1994) Noise and the neurosciences: a long history, a recent revival and some theory. In: Origins: brain and self organization (Pribram KH, ed), pp 300-331. Hillsdale, NJ: Elbaum.

Shannon EE (1949) Communication in the presence of noise. Proc IRE $37: 10-21$

Stacey WC, Durand DM (2000) Stochastic resonance improves signal detection in hippocampal CA1 neurons. J Neurophysiol 83:1394-1402.

Trulsson M (2001) Mechanoreceptive afferents in the human sural nerve. Exp Brain Res 137:111-116.

Ward LM, Neiman A, Moss F (2002) Stochastic resonance in psychophysics and in animal behavior. Biol Cybern 87:91-101.

Wiesenfeld K, Moss F (1995) Stochastic resonance and the benefits of noise: from ice ages to crayfish and SQUIDs. Nature 373:33-36.

Winterer G, Ziller M, Dorn H, Frick K, Mulert C, Dahhan N, Herrmann WM, Coppola R (1999) Cortical activation, signal-to-noise ratio and stochastic resonance during information processing in man. Clin Neurophysiol 110:1193-1203.

Yamamoto Y, Hidaka I, Iso-o N, Komai A, Soma R, Kwak S (2002) Noiseinduced compensation for postural hypotension in primary autonomic failure. Brain Res 945:71-78. 